\title{
Correction to: Clinical Decision Support
}

\section{Correction to:}

Chapter 3 in: Scottt Mankowitz (ed.), Clinical Informatics Board Review and Self Assessment, https://doi.org/10.1007/978-3-030-16230-6_3

The original version of this chapter was revised. Figure 3-8 headings was inadvertently published as Pre-test probability on both sides, now this was updated to Pre-test probability, Post-test probability.

The correction to this chapter can be found at https://doi.org/10.1007/978-3-319-63766-2_17 